\title{
Genetic control of autophagy underlies pathogenesis of inflammatory bowel disease
}

\author{
KG Lassen ${ }^{1,2}$ and RJ Xavier ${ }^{1,2,3}$
}

\begin{abstract}
Autophagy contributes to cellular homeostasis in the face of nutrient deprivation and other cellular stresses. Cell type-specific functions for autophagy are critical in maintaining homeostasis at both the tissue level and at the whole-organism level. Recent work has highlighted the ways in which human genetic variants modulate autophagy to alter epithelial and immune responses in inflammatory bowel disease.
\end{abstract}

\section{REGULATION OF AUTOPHAGY}

Autophagy is an evolutionarily conserved stress response pathway that directs cargoes to the lysosome, where they are degraded for subsequent recycling. ${ }^{1}$ Autophagy can be nonselective or selective; in the former case, autophagy functions as a bulk degradation pathway to help recycle nutrients and balance biosynthesis pathways. In contrast, nonselective autophagy involves the precise targeting of unwanted intracellular components such as protein aggregates, damaged organelles, intracellular pathogens, and lipid droplets. Autophagy is often considered a prosurvival mechanism with many benefits; however, prolonged, uncontrolled autophagy can lead to a type of cell death termed autosis. ${ }^{2}$ Therefore, the induction of autophagy and sustained autophagy must be regulated through both transcriptional and post-translational mechanisms. ${ }^{3-8}$ Emerging data have highlighted the multistep regulation of post-translational modifications in controlling autophagy, including ubiquitination, phosphorylation, and acetylation. ${ }^{9}$ These post-translational modifications are also critical in the induction of selective autophagy as well as cargo selection.

More than 30 core autophagy proteins mediate the autophagic cascade, which controls the preinitiation, initiation, and elongation of the autophagosome. ${ }^{10,11}$ Autophagy is first triggered by a preinitiation complex composed of ULK1/2, ATG13, and FIP200, which together activate an initiation complex consisting of ATG14, Beclin 1, VPS34, and VPS15 to generate the isolation membrane of a nascent autophagosome. Next, two ubiquitin-like ATG conjugation systems
(ATG12 and LC3/ATG8) control elongation of the isolation membrane into a double membrane-bound autophagosome containing cargo for degradation. Once elongation is complete, SNAREs such as syntaxin 17 mediate the fusion of autophagosomes and lysosomes, where lysosomal enzymes degrade the autophagosomal cargo. ${ }^{12-14}$ Until recently, it was widely accepted that genetic deletion or loss of any of the core ATG genes abolishes autophagosome formation and autophagic function. However, Tsuboyama et al. ${ }^{15}$ recently demonstrated using live imaging that the ATG conjugation machinery is not essential for autolysosome formation. ${ }^{15}$ Additionally, Nguyen et al. ${ }^{16}$ recently demonstrated that LC3s and GABARAPs are not required for autophagosome formation. These recent findings create a new paradigm for our understanding of the role of individual autophagy proteins in the autophagic cascade and prompt the re-evaluation of published data involving deletion of a single $A T G$ gene.

New insights by Tsuboyama et al. ${ }^{15}$ could also be useful in understanding the contribution of individual ATG proteins to autophagy as well as their roles in noncanonical autophagy and autophagy-independent pathways that are important for disease. Noncanonical autophagy has been shown to be critical in limiting inflammation in response to fungal pathogens and dying cells as well as in the response to viral and parasitic pathogens, ${ }^{17-20}$ including LC3-associated phagocytosis (LAP). In LAP, specific components of the autophagy machinery conjugate LC3 to phagosomal membranes to aid in the clearance of phagocytosed fungal pathogens. ${ }^{21,22}$ Cargoes degraded by LAP are engulfed in a single phagosomal

${ }^{1}$ Broad Institute, Cambridge, Massachusetts, USA. ${ }^{2}$ Center for Computational and Integrative Biology, Massachusetts General Hospital, Boston, Massachusetts, USA and ${ }^{3}$ Gastrointestinal Unit and Center for the Study of Inflammatory Bowel Disease, Massachusetts General Hospital, Harvard Medical School, Boston, Massachusetts, USA. Correspondence: KG Lassen (Klassen@broadinstitute.org) or RJ Xavier (xavier@molbio.mgh.harvard.edu) 
membrane that requires the protein rubicon but does not require the autophagy preinitiation complex, thus distinguishing LAP from canonical autophagy. ${ }^{21,22}$ In mice, defects in LAP lead to an autoimmune-like response to dying cells and uncontrolled inflammation. ${ }^{18}$ Additionally, LRRK2, a gene associated with Parkinson's disease, Crohn's disease, and leprosy has recently been shown to negatively regulate a noncanonical autophagy pathway, suggesting that dysregulation of this pathway may directly influence disease susceptibility. ${ }^{23}$

Recent studies have also highlighted the autophagy-independent roles of $A T G$ genes. ${ }^{20}$ For example, ULK1/2 is able to phosphorylate SEC16A and thus directly regulate ER export. ${ }^{24,25}$ Additionally, ATG5 has a unique role in the cell-specific response to Mycobacterium tuberculosis infection, although the underlying mechanism is yet to be defined. ${ }^{26}$ These data highlight how specific stimuli can regulate noncanonical autophagy and, in turn, how key autophagy genes likely have many unrecognized roles in regulating immunity.

\section{AN OVERVIEW OF AUTOPHAGY IN THE INTESTINE}

The intestine must contend with constant stress, including changes in nutrients, microbial interactions, and intestinal injury. The regulation of nutrient absorption, fluid, and ion balance requires tight control of a number of intracellular processes. Nutrient and ion levels fluctuate with nutrient intake, and control of autophagy is one way cells rapidly adjust to these fluctuations. For example, studies have shown that decreased levels of vitamin $D$ receptor or vitamin $D$ can lead to decreased levels of ATG16L1, resulting in impaired autophagy and dysbiosis. ${ }^{27,28}$ Impaired autophagy in the setting of aminoacid starvation can trigger intestinal inflammation through inflammasome activation and production of reactive oxygen species. ${ }^{29}$ Therefore, maintaining intestinal homeostasis is largely a function of controlling stress responses so as to avoid deleterious inflammation while limiting pathogenic outcomes. Autophagy is therefore required for intestinal homeostasis, the response to intestinal injury, and repair.

An additional major stress for epithelial cells and immune cells in gut tissue is the need for these cells to constantly adjust to the close juxtaposition of a large load of microbes. A critical feature of intestinal homeostasis is that cells must maintain tolerance to harmless microbes while retaining the capacity to break this tolerance to mount an effective immune response to pathogenic microbes. To accomplish this balance, gut-resident immune cells are primed for a quick response, resulting in a level of low-grade inflammation at all times. Autophagy functions at multiple steps in this process, including microbial monitoring, maintaining epithelial barrier integrity, direct intracellular pathogen clearance, and regulating inflammation. ${ }^{30,31}$ In turn, microbes and microbial products can directly alter the intestinal ecosystem and can directly influence autophagy. ${ }^{32-37}$

Autophagy is also important in controlling the development, differentiation, and function of immune subsets in the intestine.
Numerous studies have carefully dissected the contribution of autophagy pathways to intestinal T-cell development and function. ${ }^{30,38}$ Recent studies have also evaluated the relationship between autophagy and metabolism to understand how metabolic changes influence T-cell survival. ${ }^{39,40}$ It is clear that defects in autophagy preferentially disrupt regulatory T-cell development and function in the intestine. ${ }^{39,40}$ In B cells, autophagy is required for plasma cell function and antibody responses. ${ }^{41,42}$ In dendritic cells, autophagy controls how antigens are processed and presented for antigen presentation. ${ }^{43}$ In macrophages, autophagy limits inflammasomemediated cytokine production and controls LAP-mediated elimination of pathogens. ${ }^{44}$ Innate lymphoid cells are groups of innate cells that orchestrate cellular crosstalk in the intestine and other tissues to modulate inflammatory responses. ${ }^{45}$ It has been recently demonstrated that autophagy is required for innate lymphoid cell development and function. ${ }^{46}$ Taken together, these data highlight the fundamental role for autophagy in balancing immunity and promoting survival of key immune cells during the immune response (Figure 1).

Selective autophagy is critical in the response to pathogens as well as in cellular homeostasis. Numerous forms of selective autophagy have been identified that target infectious microbes such as bacteria (anti-bacterial autophagy), viruses (virophagy), and parasites (parasitophagy) as well as damaged or malfunctioning organelles such as lysosomes (lysophagy), endoplasmic reticulum (ERophagy), endosomes, and mitochondria (mitophagy). ${ }^{47}$ Additionally, autophagy-mediated turnover of lipids (lipophagy), aggregated protein (aggrephagy), and ferritin (ferritinophagy) have also been shown to be important in maintaining cellular physiology, and these pathways might have important cell type-specific consequences in the gut. ${ }^{48,49}$ For example, lysophagy is involved in sensing and clearing membrane damage to limit injury. ${ }^{50,51}$ Receptors for these processes have been recently identified; however, there are likely a multitude of receptors for selective autophagy to create substrate selectivity and many receptors remain to be discovered. ${ }^{47,48,52-54}$

\section{AUTOPHAGY IN RESPONSE TO EPITHELIAL INJURY}

Epithelial cells in the intestine form a physical barrier between the host and the luminal environment. Cellular junction proteins control paracellular permeability, allowing macromolecules such as nutrients to pass but limiting the dissemination of microbes. ${ }^{55}$ Autophagy impinges upon many regulatory steps in maintaining barrier function, including cell typespecific functions. Epithelial barrier integrity can be directly controlled by autophagy through the degradation of the tight junction protein claudin-2. ${ }^{56}$ In Caenorhabditis elegans, bacterial pore-forming toxin stimulates the transcription factor HLH-30 (TFEB), which induces autophagy, lysosomal, and inflammatory genes to control bacterial infection and maintain host tolerance. ${ }^{57,58}$ Both junctional integrity and the direct clearance of bacteria through selective autophagy are considered to be the mechanisms underlying this control. 

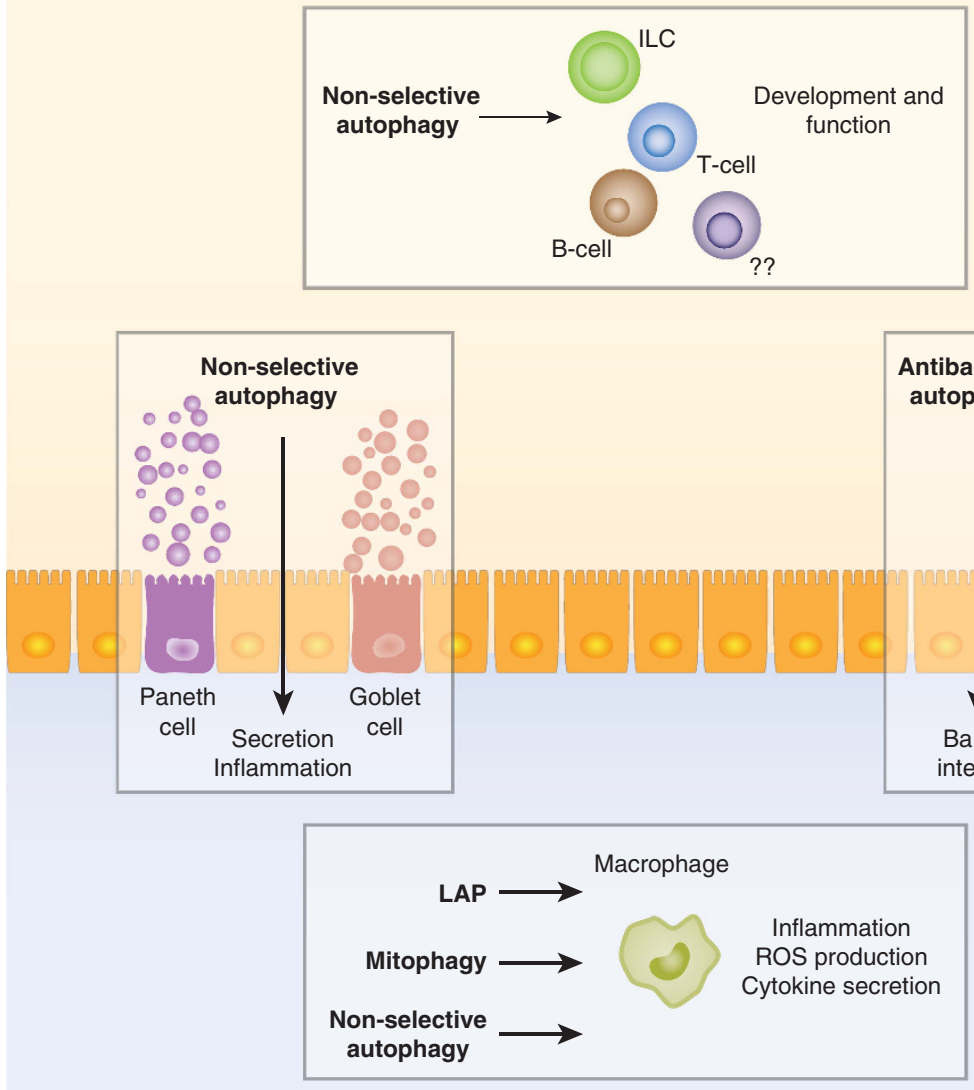

Figure 1 Pleiotropic effects of autophagy in the intestine. Nonselective or bulk autophagy underlies cellular homeostasis in the intestine. Additionally, perturbations to noncanonical autophagy and cargo-selective autophagy result in cell type-specific alterations. This schematic illustrates how disruptions to autophagy, selective autophagy, or noncanonical autophagy can impact intestinal epithelial cells as well as both innate and adaptive immune cells. Furthermore, it is likely that autophagy controls the differentiation of rare cell types that have yet to be defined.

Autophagy therefore has a role in tissue remodeling, immunity, and inflammation.

Autophagy is also critical for the cell type-specific control of specialized secretory intestinal epithelial cells, including Paneth cells and goblet cells, which together enforce the barrier between intestinal epithelial cells and the commensal microbiota. Paneth cells are located at the base of the crypts in the small intestine and contain large secretory granules that secrete large quantities of anti-microbial peptides. Goblet cells secrete mucins, which form the mucous layer in the intestine. Defects in autophagy affect the development and function of both of these cell types: for example, defects in autophagy disrupt secretary granule morphology and secretion in both Paneth cells and goblet cells, resulting in changes in the inflammatory microenvironment. ${ }^{59-62}$ Autophagy mediates polarized secretion of cytokines and lysosomal contents in highly secretory cell types, suggesting that autophagy controls common vulnerabilities in these cell types. ${ }^{63,64}$ The precise links between degradative autophagy and autophagy-mediated control of secretion are still being defined, although recent reports suggest specialized cargo receptors and SNAREs are involved. ${ }^{65}$

\section{AUTOPHAGY AND THE HOST RESPONSE TO BACTERIA}

Core autophagy genes in intestinal epithelial cells are central to the control of the dissemination of Salmonella enterica serovar Typhimurium infection in vivo. ${ }^{66,67}$ Anti-bacterial autophagy represents an important defense mechanism against the intracellular pathogens S. Typhimurium, Shigella flexneri, and Listeria monocytogenes. To trigger anti-bacterial autophagy, intracellular bacteria must be sensed and then marked for degradation by post-translational modifications and/or recruitment of adaptor proteins such as NDP52, p62, optineurin, and NBR1. ${ }^{68}$ These adaptors bring marked cargo into close proximity with the autophagy machinery through LC3interacting region motifs, which mediate the interaction with ATG8 family proteins. Some intracellular bacteria express type III secretion systems that allow bacteria to escape into the cytosol, triggering membrane damage resulting in the exposure of host glycan "eat me" signals. These signals recruit galectin-8 and TBK1, which in turn can recruit autophagy adapter proteins. ${ }^{69,70}$ Ubiquitination has a key role in bacterial targeting and a role has recently been emerging for specific E3 ligases in modulating substrate specificity in the autophagy pathway. ${ }^{71,72}$ Several E3 ubiquitin ligases including LRSAM1, Parkin, 
SMURF1, RNF166, and some TRIM proteins have each been shown to ubiquitinate bacteria or bacteria-associated proteins to drive anti-bacterial autophagy. ${ }^{73-76}$ Various TRIM proteins have also been implicated in anti-bacterial autophagy as well as other types of selective autophagy that have a direct effect on inflammation. $^{74,77,78}$

Bacteria have evolved several mechanisms to cope with the selective pressure of host autophagy, underscoring the importance of autophagy in bacterial defense. Typically, these mechanisms involve the expression of bacterial proteins that mediate escape from the autophagy machinery or evasion from bacterial targeting. ${ }^{79-81}$ Some pathogens have even evolved ways to exploit the autophagy machinery for bacterial growth. Bhogaraju et al..$^{82}$ recently reported a unique evasion mechanism by Legionella pneumophila to suppress autophagy. In this process, a L. pneumophila effector protein promotes arginine phosphoribosylation of ubiquitin, resulting in a disruption of autophagy as well as other ubiquitin-dependent cellular processes. Taken together, these studies highlight the multilayered defense network that serves to target bacteria for degradation to protect the host in the event of bacterial evolution and escape.

\section{CROSS-REGULATION OF SELECTIVE AUTOPHAGY PATHWAYS}

Although core autophagy proteins are highly conserved, there is much functional diversity in proteins involved in the targeting and recognition of selective autophagy substrates such as damaged mitochondria or pathogens including bacteria and viruses. Mitophagy is a selective form of autophagy that eliminates damaged mitochondria or helps to reduce the number of mitochondria when cellular energy demands are low. ${ }^{83}$ Mitochondria function to boost innate responses to bacteria and viruses through generation of reactive oxygen species and by serving as key scaffolds for some pattern recognition receptors in immune cells. In the intestine of C. elegans, mitochondrial damage triggers mitophagy through an innate immune pathway; this pathway is neuroprotective, demonstrating that effects of mitophagy are not cell autonomous. ${ }^{84}$ Recent studies have identified a number of proteins that function in mitophagy, anti-bacterial autophagy, and/or virophagy, this definition was already given earlier in the review suggesting that these processes share signaling components. The E3 ubiquitin ligase Parkin is critical for mitophagy and has recently been shown to be recruited to intracellular $M$. tuberculosis and S. Typhimurium to promote autophagy. ${ }^{54,74,85}$ The E3 ligase SMURF1 and the peroxisomal protein PEX13 are also critical for mitophagy, virophagy, and anti-bacterial autophagy. ${ }^{76,86,87}$ The kinase TBK1 has also recently been shown to be actively recruited to bacteria and damaged mitochondria; once there, TBK1dependent phosphorylation recruits downstream adaptors required for anti-bacterial autophagy and mitophagy. ${ }^{53,69}$ These data highlight the strong overlap between xenophagy and mitophagy.

\section{AUTOPHAGY AND CELLULAR STRESS RESPONSES}

Recent data have revealed strong connections between autophagy and other stress response pathways such as reactive oxygen species, antioxidant response, DNA repair, ER stress, and inflammation. The adaptor protein p62 is recruited to defective mitochondria and intracellular bacteria and is required for Parkin-dependent mitophagy and pathogenspecific anti-bacterial autophagy. ${ }^{74,88}$ The NRF2-KEAP1 pathway upregulates anti-inflammatory and cytoprotective genes to protect from oxidative stress. ${ }^{89}$ Recently, p62 has been shown to link autophagy with the NRF2-KEAP1 pathway by mediating selective autophagy of KEAP1, thereby promoting NRF2 activation. ${ }^{90}$ This finding suggests a mechanism whereby selective autophagy increases a secondary protective stress response pathway.

A recent study demonstrated that the Fanconi anemia complementation group $\mathrm{C}$ protein interacts with Parkin to execute mitophagy, and loss of Fanconi anemia complementation group $\mathrm{C}$ increases interleukin-1 $\beta$ (IL-1 $\beta$ ) and reactive oxygen species production. ${ }^{91}$ This finding is consistent with studies showing that defective mitophagy directly contributes to increased IL-1 $\beta$ secretion. ${ }^{92}$ Taken together, these data suggest that impaired selective autophagy in the intestine disrupts direct bacterial clearance as well as mitophagy, with direct implications for inflammation.

Significant attention has also been given to the interplay between ER stress and autophagy in the intestine. ${ }^{93}$ Paneth cell-specific deletion of $X b p 1$, which encodes a transcription factor that controls the unfolded protein response, is sufficient to induce Crohn's disease-like ileitis. ${ }^{94}$ Additionally, ER stress and autophagy were shown to function as compensatory pathways in intestinal epithelial cells; loss of both pathways resulted in more severe disease. ${ }^{94}$ These data underscore the critical role of stress response pathways in the intestine and highlight the crucial role for autophagy in coordinating these responses.

\section{AUTOPHAGY AND INFLAMMATORY BOWEL DISEASE}

Inflammatory bowel disease (IBD) is a complex disease involving chronic inflammation of the gastrointestinal tract, including Crohn's disease and ulcerative colitis. Over the past decade, there has been enormous progress in the discovery of numerous genetic variants that increase or decrease the risk of IBD. These genetic variants have provided a roadmap to understanding key cell types and pathways controlling intestinal homeostasis. ${ }^{95-99}$ The connection between autophagy and IBD was first realized through the identification of a common coding polymorphism in the core autophagy gene ATG16L1 (ATG16L1 T300A) that confers increased risk of Crohn's disease. ${ }^{98,100}$ Since this discovery, other genes associated with Crohn's disease susceptibility, including IRGM, have also been associated with anti-bacterial autophagy and the clearance of intracellular pathogens, underscoring the importance of this pathway in IBD. ${ }^{101}$

Multiple studies have investigated the contribution of autophagy to IBD susceptibility and chronic disease. ${ }^{102}$ Studies 
of Crohn's disease patients who express the ATG16L1 T300A polymorphism have demonstrated increases in proinflammatory cytokines in response to specific stimuli. ${ }^{103}$ In addition, defects in the size and number of Paneth cell granules are also found in patients harboring ATG16L1 T300A, suggesting that these cell types are highly susceptible to perturbations in autophagy and highlighting the role for autophagy in Paneth cell development and function. ${ }^{104}$ In patients with quiescent disease, Paneth cells exhibit higher levels of ER stress when the ATG16L1 T300A polymorphism is present, further supporting a role for both ER stress and autophagy defects in disease pathogenesis. ${ }^{105,106}$ Additionally, recent studies suggest that accumulation of IRE1 $\alpha$ in autophagy-deficient mice and in individuals homozygous for the T300A polymorphism promotes intestinal inflammation. ${ }^{107}$

Studies of ATG16L1 T300A mice have shown that this polymorphism confers impaired anti-bacterial autophagy and defects in Paneth cells and goblet cells similar to those reported in human studies. ${ }^{60,61}$ Recent studies have also found that microbiome-derived outer membrane vesicles, which are often shed in the intestinal lumen, are inefficient at inducing regulatory $\mathrm{T}$ cells to suppress inflammation and promote microbial tolerance in mice and humans that harbor the ATG16L1 T300A polymorphism. ${ }^{108}$ Finally, ATG16L1 T300A has also been shown to control dendritic cell migration through RAC1 hyperactivation. ${ }^{109}$ Thus, a single-nucleotide polymorphism in a core autophagy gene can exert cell type-specific effects as well as alterations to host-microbe dynamics, and these effects can cooperate to exacerbate inflammation and impair anti-bacterial responses.

The mechanistic basis for disruption of autophagy by ATG16L1 T300A was found to be an increase in caspase 3mediated cleavage of ATG16L1 T300A, resulting in decreased protein levels and disruption of selective autophagy. ${ }^{60,61}$ Additionally, the T300A polymorphism has been shown to disrupt unconventional autophagy mediated by TMEM59. ${ }^{110}$
Calpain-mediated cleavage of ATG5 and Beclin 1 has also been shown to be increased in patients with colitis, suggesting that autophagy protein stability in the intestine may underlie the inflammation that contributes to IBD. ${ }^{111}$ Finally, recent studies have identified a role for IKK $\alpha$ in the phosphorylation and stabilization of ATG16L1 to prevent caspase-dependent cleavage in response to extracellular stimuli, further highlighting the importance of ATG16L1 stabilization during inflammation. ${ }^{112}$

Additional genes within IBD susceptibility loci have also recently been shown to control autophagy. The MTMR3 IBD risk allele decreases pattern recognition receptor-mediated autophagy, thereby increasing IL-1 $\beta$ secretion and nuclear factor- $\kappa B$ signaling. ${ }^{113}$ Moreover, a small interfering RNA screen of IBD-associated genes in epithelial cells found 31 IBDassociated genes that function in anti-bacterial autophagy (as measured by LC3-bacteria colocalization) or intracellular pathogen defense (as measured by intracellular bacterial replication). The discovery of autophagy-dependent roles for these genes in bacterial defense strengthens the critical role for autophagy in IBD and identifies important new functionally related pathways that likely contribute to pathogenesis. As discussed above, this study identified roles for IBD risk genes PEX13 and SMURF1 in anti-bacterial autophagy, expanding their function beyond mitophagy. ${ }^{87}$ PEX13 may have autophagy-independent roles in pathogen defense, as infection has been shown to induce innate signaling from peroxisomes. ${ }^{114}$ RUFY4 positively regulates autophagy in response to IL-4, indicating the relationship between immune activation and autophagy stimulation. ${ }^{115} \mathrm{C} 1$ orf106, a protein of unknown function encoded by an IBD risk gene, was shown in large-scale binding data sets to associate with NDP52 as well as TRIM27, an E3 ligase that regulates degradation of NOD2, suggesting a potential mechanism for a role in anti-bacterial autophagy. ${ }^{116,117}$ Finally, the IBD risk gene SLC39A8 encodes a solute carrier that transports manganese and zinc into cells as

Table 1 Genes within IBD risk loci that function in autophagy-related pathways and bacterial defense

\begin{tabular}{ll}
\hline Gene & Function in autophagy-related pathways or bacterial defense \\
\hline GPR65 & Autophagy and lysosomal function \\
SMURF1 & Mitophagy, virophagy, anti-bacterial autophagy \\
PEX13 & Mitophagy, virophagy, anti-bacterial autophagy \\
RUFY4 & Autophagy and endosomal trafficking in immune cells \\
C10rf106 & Interacts with NDP52, function unknown \\
UTS2 & UTS2R ligation by UTS2 impairs autophagosome formation \\
TPPP & Promotes unconventional autophagy-dependent secretion \\
UBA7 & Inhibits autophagy through ISGylation \\
SLC22A4 & Upregulated in response to infection with Shigella in vivo \\
LITAF & Upregulates autophagy \\
ATG16L1 & Core autophagy gene \\
IRGM & Anti-bacterial autophagy \\
\hline
\end{tabular}

Abbreviation: IBD, Inflammatory bowel disease. 
an electroneutral metal/bicarbonate symporter. ${ }^{118}$ The anti-bacterial autophagy adapter proteins NDP52, p62, optineurin, and NBR1 each contain a zinc-finger motif that requires coordination of a zinc ion, and this domain in NDP52 is required for ubiquitin binding and recognition. ${ }^{69,119,120} \mathrm{It}$ is tempting to speculate that impaired zinc transport associated with polymorphisms in SLC39A8 might alter anti-bacterial autophagy with direct consequences on microbial composition. ${ }^{121,122}$ These genes as well as others are summarized in Table 1. These findings underscore the role of autophagy in intestinal homeostasis and highlight the myriad pathways that converge to perturb autophagy.

Recent studies also elucidated a role for GPR65, a $\mathrm{H}^{+}$sensing G-protein-coupled receptor, in maintaining lysosomal function. ${ }^{87,131}$ A number of IBD-associated polymorphisms are located within the GPR65 locus, including one polymorphism, $\mathrm{I} 231 \mathrm{~L}$, that results in a missense mutation. Loss of GPR65 or expression of GPR65 I231L resulted in increased lysosomal pH, impaired anti-bacterial autophagy, and accumulation of aberrant lysosomes. ${ }^{87}$ Gpr $65^{-1-}$ mice were more susceptible to the murine intestinal pathogen Citrobacter rodentium, failing to clear the infection and exhibiting more severe epithelial damage and increased inflammatory cell recruitment. ${ }^{87}$ Taken together, these data underscore the role of GPR65 in lysosomal biology and elucidate the lysosome as a critical regulator of immune function and epithelial homeostasis in the intestinal environment.

Expression of GPR65 is largely restricted to the intestine and immune cells, allowing a distinction between patients with lysosomal storage diseases due to mutations in broadly expressed lysosomal genes in contrast to patients with GPR65 polymorphisms that reduce GPR65 function. Hermansky-Pudlak syndrome is a rare disorder resulting from mutations in genes involved in the formation and trafficking of lysosome-related organelles, and a subset of patients with Hermansky-Pudlak syndrome develop Crohn's disease-like colitis. ${ }^{132}$ A subset of patients with Niemann-Pick disease type $\mathrm{C} 1$, a lysosomal lipid storage disorder caused by mutations in NPC1, also develop Crohn's disease-like symptoms. ${ }^{133}$ Impaired anti-bacterial autophagy is also observed with mutations in NPC1. ${ }^{133}$ These data suggest that changes in lysosomal function, particularly in immune cells or intestinal epithelial cells, can alter susceptibility to microbes at the hostcommensal interface. Additionally, inflammatory signaling can be amplified when endosomes or phagosomes formed after microbial recognition are not properly cleared through the lysosome, providing another mechanism for sustaining a proinflammatory feedback loop in the setting of microbial stimuli. $^{134}$

\section{PERSPECTIVES}

Dysregulation of autophagy contributes to IBD susceptibility. ${ }^{135}$ Recent studies on the roles of Zellweger syndrome spectrum disorder gene PEX13 and the Joubert syndrome gene INPP5E in autophagy suggest that autophagy dysregulation may underlie a number of as yet unrecognized diseases. ${ }^{136,137}$ Disruption of autophagy can also directly contribute to uncontrolled inflammation. These data suggest that small molecules that induce autophagy may hold broad therapeutic potential. However, the cross-regulation of autophagy and other cellular stress response pathways make selective targeting of autophagy challenging in vivo. Additionally, more mechanistic insights into the pathways that contribute to disease will be necessary. In the case of patients with the GPR65 I231L polymorphism, a robust autophagy inducer may exacerbate disease and only serve to increase the burden on poorly functional lysosomes. There is an urgent need for better tools to study primary and secondary effects of novel genes and small molecules on autophagy. Recent studies have described new methods to more precisely evaluate autophagic flux and to study the effects of ATG gene deletion in multiple cell types in vivo. ${ }^{138,139}$ These tools will be helpful in elucidating the autophagy-dependent effects of genes and small molecules in the control of intestinal homeostasis and IBD.

\section{ACKNOWLEDGMENTS}

We thank Natalia Nedelsky for editorial assistance. This work was supported by the Helmsley Charitable Trust.

\section{AUTHOR CONTRIBUTIONS}

K.L. and R.J.X. wrote the article.

\section{DISCLOSURE}

The authors declared no conflict of interest.

(c) 2017 Society for Mucosal Immunology

\section{REFERENCES}

1. Levine, B. \& Klionsky, D.J. Autophagy wins the 2016 Nobel Prize in Physiology or Medicine: Breakthroughs in baker's yeast fuel advances in biomedical research. Proc. Natl Acad. Sci. USA 114, 201-205 (2016).

2. Liu, $\mathrm{Y}$. et al. Autosis is a $\mathrm{Na}+, \mathrm{K}+-$ ATPase-regulated form of cell death triggered by autophagy-inducing peptides, starvation, and hypoxiaischemia. Proc. Natl Acad. Sci. USA 110, 20364-20371 (2013).

3. Nazio, F. et al. Fine-tuning of ULK1 mRNA and protein levels is required for autophagy oscillation. J. Cell Biol. 215, 841-856 (2016).

4. Bernard, A., Jin, M., Xu, Z. \& Klionsky, D.J. A large-scale analysis of autophagy-related gene expression identifies new regulators of autophagy. Autophagy 11, 2114-2122 (2015).

5. Bernard, A. et al. Rph1/KDM4 mediates nutrient-limitation signaling that leads to the transcriptional induction of autophagy. Curr. Biol. 25, 546-555 (2015).

6. Fullgrabe, J. et al. The histone $\mathrm{H} 4$ lysine 16 acetyltransferase hMOF regulates the outcome of autophagy. Nature 500, 468-471 (2013).

7. Settembre, C. et al. TFEB links autophagy to lysosomal biogenesis. Science 332, 1429-1433 (2011)

8. Lapierre, L.R., Kumsta, C., Sandri, M., Ballabio, A. \& Hansen, M. Transcriptional and epigenetic regulation of autophagy in aging. Autophagy 11, 867-880 (2015)

9. Wani, W.Y., Boyer-Guittaut, M., Dodson, M., Chatham, J., Darley-Usmar, V. \& Zhang, J. Regulation of autophagy by protein post-translational modification. Lab. Invest. 95, 14-25 (2015).

10. Deretic, V., Saitoh, T. \& Akira, S. Autophagy in infection, inflammation and immunity. Nat. Rev. Immunol. 13, 722-737 (2013).

11. Levine, B., Mizushima, N. \& Virgin, H.W. Autophagy in immunity and inflammation. Nature 469, 323-335 (2011).

12. Itakura, E., Kishi-Itakura, C. \& Mizushima, N. The hairpin-type tail-anchored SNARE syntaxin 17 targets to autophagosomes for fusion with endosomes/lysosomes. Cell 151, 1256-1269 (2012). 
13. Diao, J. et al. ATG14 promotes membrane tethering and fusion of autophagosomes to endolysosomes. Nature 520, 563-566 (2015).

14. Nair, U. et al. SNARE proteins are required for macroautophagy. Cell $\mathbf{1 4 6}$, 290-302 (2011).

15. Tsuboyama, K., Koyama-Honda, I., Sakamaki, Y., Koike, M., Morishita, H. \& Mizushima, N. The ATG conjugation systems are important for degradation of the inner autophagosomal membrane. Science 354 , 1036-1041 (2016).

16. Nguyen, T.N., Padman, B.S., Usher, J., Oorschot, V., Ramm, G. \& Lazarou, M. Atg8 family LC3/GABARAP proteins are crucial for autophagosome-lysosome fusion but not autophagosome formation during PINK1/Parkin mitophagy and starvation. J. Cell. Biol. 215, 857-874 (2016).

17. Selleck, E.M. et al. A noncanonical autophagy pathway restricts toxoplasma gondii growth in a strain-specific manner in IFN-gammaactivated human cells. MBio 6, e01157-01115 (2015).

18. Martinez, J. et al. Noncanonical autophagy inhibits the autoinflammatory, lupus-like response to dying cells. Nature 533, 115-119 (2016).

19. Oikonomou, V. etal. Noncanonical fungal autophagy inhibits inflammation in response to iFN-gamma via DAPK1. Cell Host Microbe 20, 744-757 (2016).

20. Mauthe, M. et al. An siRNA screen for ATG protein depletion reveals the extent of the unconventional functions of the autophagy proteome in virus replication. J Cell Biol 214, 619-635 (2016).

21. Martinez, J. et al. Molecular characterization of LC3-associated phagocytosis reveals distinct roles for Rubicon, NOX2 and autophagy proteins. Nat. Cell. Biol. 17, 893-906 (2015).

22. Boyle, K.B. \& Randow, F. Rubicon swaps autophagy for LAP. Nat. Cell Biol. 17, 843-845 (2015).

23. Manzoni, C. et al. mTOR independent regulation of macroautophagy by leucine rich repeat kinase 2 via beclin-1. Sci. Rep. 6, 35106 (2016).

24. Joo, J.H. et al. The noncanonical role of ULK/ATG1 in ER-to-Golgi trafficking is essential for cellular homeostasis. Mol. Cell 62, 491-506 (2016).

25. Zhao, Y.G. \& Zhang, H. The incredible ULKs: autophagy and beyond. Mol. Cell 62, 475-476 (2016).

26. Kimmey, J.M. et al. Unique role for ATG5 in neutrophil-mediated immunopathology during $M$. tuberculosis infection. Nature 528, 565-569 (2015).

27. Jin, D. et al. Lack of vitamin D receptor causes dysbiosis and changes the functions of the murine intestinal microbiome. Clin. Ther. 37, 996-1009 e1007 (2015).

28. Wu, S. et al. Intestinal epithelial vitamin $\mathrm{D}$ receptor deletion leads to defective autophagy in colitis. Gut 64, 1082-1094 (2015).

29. Ravindran, R. et al. The amino acid sensor GCN2 controls gut inflammation by inhibiting inflammasome activation. Nature 531, 523527 (2016).

30. Kabat, A.M., Pott, J. \& Maloy, K.J. The mucosal immune system and its regulation by autophagy. Front. Immunol. 7, 240 (2016).

31. Deretic, V., Kimura, T., Timmins, G., Moseley, P., Chauhan, S. \& Mandell, M. Immunologic manifestations of autophagy. J. Clin. Invest. 125, 75-84 (2015).

32. Inaba, Y. et al. Soluble bioactive microbial mediators regulate proteasomal degradation and autophagy to protect against inflammation-induced stress. Am. J. Physiol. Gastrointest. Liver Physiol. 311, G634-G647 (2016).

33. Kishino, S. et al. Polyunsaturated fatty acid saturation by gut lactic acid bacteria affecting host lipid composition. Proc. Natl Acad. Sci. USA 110, 17808-17813 (2013).

34. Wang, C. et al. CD5L/AIM regulates lipid biosynthesis and restrains Th17 Cell pathogenicity. Cell 163, 1413-1427 (2015).

35. Macia, L. et al. Metabolite-sensing receptors GPR43 and GPR109A facilitate dietary fibre-induced gut homeostasis through regulation of the inflammasome. Nat. Commun. 6, 6734 (2015).

36. Maslowski, K.M. et al. Regulation of inflammatory responses by gut microbiota and chemoattractant receptor GPR43. Nature 461, 1282-1286 (2009).

37. Venkatesh, M. et al. Symbiotic bacterial metabolites regulate gastrointestinal barrier function via the xenobiotic sensor PXR and Toll-like receptor 4. Immunity 41, 296-310 (2014).
38. Oral, O., Yedier, O., Kilic, S. \& Gozuacik, D. Involvement of autophagy in T cell biology. Histol. Histopathol. 32, 11-20 (2017).

39. Wei, J. et al. Autophagy enforces functional integrity of regulatory Tcells by coupling environmental cues and metabolic homeostasis. Nat. Immunol. 17, 277-285 (2016).

40. Kabat, A.M. etal. The autophagy gene Atg16l1 differentially regulates Treg and TH2 cells to control intestinal inflammation. Elife 5, e12444 (2016).

41. Conway, K.L. et al. ATG5 regulates plasma cell differentiation. Autophagy 9, 528-537 (2013).

42. Pengo, N. et al. Plasma cells require autophagy for sustainable immunoglobulin production. Nat. Immunol. 14, 298-305 (2013).

43. Munz, C. Autophagy beyond intracellular MHC class II antigen presentation. Trends Immunol. 37, 755-763 (2016).

44. Saitoh, T. \& Akira, S. Regulation of inflammasomes by autophagy. J. Allergy Clin. Immunol. 138, 28-36 (2016).

45. Artis, D. \& Spits, H. The biology of innate lymphoid cells. Nature $\mathbf{5 1 7}$, 293-301 (2015).

46. O'Sullivan, T.E. et al. Atg5 is essential for the development and survival of innate lymphocytes. Cell Rep. 15, 1910-1919 (2016).

47. Khaminets, A. et al. Regulation of endoplasmic reticulum turnover by selective autophagy. Nature 522, 354-358 (2015).

48. Mancias, J.D., Wang, X., Gygi, S.P., Harper, J.W. \& Kimmelman, A.C. Quantitative proteomics identifies NCOA4 as the cargo receptor mediating ferritinophagy. Nature 509, 105-109 (2014).

49. Singh, R. et al. Autophagy regulates lipid metabolism. Nature 458 , 1131-1135 (2009).

50. Maejima, I. et al. Autophagy sequesters damaged lysosomes to control lysosomal biogenesis and kidney injury. EMBO J 32, 2336-2347 (2013).

51. Chauhan, S. et al. TRIMs and galectins globally cooperate and TRIM16 and galectin-3 co-direct autophagy in endomembrane damage homeostasis. Dev. Cell 39, 13-27 (2016).

52. McEwan, D.G. et al. PLEKHM1 regulates autophagosome-lysosome fusion through HOPS complex and LC3/GABARAP proteins. Mol. Cell 57, 39-54 (2015).

53. Richter, B. et al. Phosphorylation of OPTN by TBK1 enhances its binding to $\mathrm{Ub}$ chains and promotes selective autophagy of damaged mitochondria. Proc. Natl Acad. Sci. USA 113, 4039-4044 (2016).

54. Lazarou, M. et al. The ubiquitin kinase PINK1 recruits autophagy receptors to induce mitophagy. Nature 524, 309-314 (2015).

55. Ramanan, D. \& Cadwell, K. Intrinsic defense mechanisms of the intestinal epithelium. Cell Host Microbe 19, 434-441 (2016).

56. Nighot, P.K., Hu, C.A. \& Ma, T.Y. Autophagy enhances intestinal epithelial tight junction barrier function by targeting claudin-2 protein degradation. J. Biol. Chem. 290, 7234-7246 (2015).

57. Chen, H.D. et al. HLH-30/TFEB-mediated autophagy functions in a cellautonomous manner for epithelium intrinsic cellular defense against bacterial pore-forming toxin in C. elegans. Autophagy 13, 371-385 (2017).

58. Visvikis, O. et al. Innate host defense requires TFEB-mediated transcription of cytoprotective and antimicrobial genes. Immunity 40, 896-909 (2014).

59. Cadwell, K. et al. Virus-plus-susceptibility gene interaction determines Crohn's disease gene Atg16L1 phenotypes in intestine. Cell 141, 1135-1145 (2010).

60. Lassen, K.G. etal. Atg16L1 T300A variant decreases selective autophagy resulting in altered cytokine signaling and decreased antibacterial defense. Proc. Natl Acad. Sci. USA 111, 7741-7746 (2014).

61. Murthy, A. et al. A Crohn's disease variant in Atg16/1 enhances its degradation by caspase 3. Nature 506, 456-462 (2014).

62. Wlodarska, M. et al. NLRP6 inflammasome orchestrates the colonic host-microbial interface by regulating goblet cell mucus secretion. Cell 156, 1045-1059 (2014).

63. DeSelm, C.J. et al. Autophagy proteins regulate the secretory component of osteoclastic bone resorption. DevCell 21, 966-974 (2011).

64. Dupont, N., Jiang, S., Pilli, M., Ornatowski, W., Bhattacharya, D. \& Deretic, V. Autophagy-based unconventional secretory pathway for extracellular delivery of IL-1beta. EMBO J 30, 4701-4711 (2011).

65. Kimura, T. et al. Dedicated SNAREs and specialized TRIM cargo receptors mediate secretory autophagy. EMBO J. 36, 42-60 (2016). 
66. Conway, K.L. et al. Atg16l1 is required for autophagy in intestinal epithelial cells and protection of mice from Salmonella infection. Gastroenterology 145, 1347-1357 (2013).

67. Benjamin, J.L., Sumpter, R. Jr., Levine, B. \& Hooper, L.V. Intestinal epithelial autophagy is essential for host defense against invasive bacteria. Cell Host Microbe 13, 723-734 (2013).

68. Sorbara, M.T. \& Girardin, S.E. Emerging themes in bacterial autophagy. Curr. Opin. Microbiol. 23, 163-170 (2015).

69. Thurston, T.L. et al. Recruitment of TBK1 to cytosol-invading Salmonella induces WIPI2-dependent antibacterial autophagy. EMBO J 35, 1779-1792 (2016).

70. Thurston, T.L., Wandel, M.P., von Muhlinen, N., Foeglein, A. \& Randow, F. Galectin 8 targets damaged vesicles for autophagy to defend cells against bacterial invasion. Nature 482, 414-418 (2012).

71. Khaminets, A., Behl, C. \& Dikic, I. Ubiquitin-dependent and independent signals in selective autophagy. Trends Cell Biol. 26, 6-16 (2016).

72. Fiskin, E., Bionda, T., Dikic, I. \& Behrends, C. Global analysis of host and bacterial ubiquitinome in response to Salmonella Typhimurium infection. Mol. Cell 62, 967-981 (2016).

73. Huett, A. et al. The LRR and RING domain protein LRSAM1 is an E3 ligase crucial for ubiquitin-dependent autophagy of intracellular Salmonella Typhimurium. Cell Host Microbe 12, 778-790 (2012).

74. Heath, R.J. et al. RNF166 determines recruitment of adaptor proteins during antibacterial autophagy. Cell Rep. 17, 2183-2194 (2016).

75. Manzanillo, P.S. et al. The ubiquitin ligase parkin mediates resistance to intracellular pathogens. Nature 501, 512-516 (2013).

76. Franco, L.H. et al. The ubiquitin ligase smurf1 functions in selective autophagy of Mycobacterium tuberculosis and anti-tuberculous host defense. Cell Host Microbe 21, 59-72 (2016).

77. Kimura, T. et al. TRIM-mediated precision autophagy targets cytoplasmic regulators of innate immunity. J. Cell Biol. 210, 973-989 (2015).

78. Kimura, T., Mandell, M. \& Deretic, V. Precision autophagy directed by receptor regulators-emerging examples within the TRIM family. J. Cell Sci. 129, 881-891 (2016).

79. Sherwood, R.K. \& Roy, C.R. Autophagy evasion and endoplasmic reticulum subversion: the Yin and Yang of Legionella intracellular infection. Annu. Rev. Microbiol. 70, 413-433 (2016).

80. Huang, J. \& Brumell, J.H. Bacteria-autophagy interplay: a battle for survival. Nat. Rev. Microbiol. 12, 101-114 (2014).

81. Kohler, L.J. \& Roy, C.R. Autophagic targeting and avoidance in intracellular bacterial infections. Curr. Opin. Microbiol. 35, 36-41 (2016).

82. Bhogaraju, S. et al. Phosphoribosylation of ubiquitin promotes serine ubiquitination and impairs conventional ubiquitination. Cell 167, 1636-1649 e1613 (2016).

83. Randow, F. \& Youle, R.J. Self and nonself: how autophagy targets mitochondria and bacteria. Cell Host Microbe 15, 403-411 (2014).

84. Chikka, M.R., Anbalagan, C., Dvorak, K., Dombeck, K. \& Prahlad, V. The mitochondria-regulated immune pathway activated in the $C$. elegans intestine is neuroprotective. Cell Rep. 16, 2399-2414 (2016).

85. Youle, R.J. \& Narendra, D.P. Mechanisms of mitophagy. Nat. Rev. Mol. Cell. Biol. 12, 9-14 (2011).

86. Orvedahl, A. et al. Image-based genome-wide siRNA screen identifies selective autophagy factors. Nature 480, 113-117 (2011).

87. Lassen, K.G. et al. Genetic coding variant in GPR65 alters lysosomal pH and links lysosomal dysfunction with colitis risk. Immunity 44, 1392-1405 (2016).

88. Geisler, S. et al. PINK1/Parkin-mediated mitophagy is dependent on VDAC1 and p62/SQSTM1. Nat. Cell Biol. 12, 119-131 (2010).

89. Jiang, T., Harder, B., Rojo de la Vega, M., Wong, P.K., Chapman, E. \& Zhang, D.D. P62 links autophagy and Nrf2 signaling. Free Radic. Biol. Med. 88 (Part B), 199-204 (2015).

90. Ichimura, Y. et al. Phosphorylation of p62 activates the Keap1-Nrf2 pathway during selective autophagy. MolCell 51, 618-631 (2013).

91. Sumpter, R. Jr et al. Fanconi anemia proteins function in mitophagy and immunity. Cell 165, 867-881 (2016).

92. Nakahira, K. et al. Autophagy proteins regulate innate immune responses by inhibiting the release of mitochondrial DNA mediated by the NALP3 inflammasome. Nat. Immunol. 12, 222-230 (2011).
93. Kaser, A. et al. XBP1 links ER stress to intestinal inflammation and confers genetic risk for human inflammatory bowel disease. Cell 134, 743-756 (2008).

94. Adolph, T.E. et al. Paneth cells as a site of origin for intestinal inflammation. Nature 503, 272-276 (2013).

95. Jostins, L. et al. Host-microbe interactions have shaped the genetic architecture of inflammatory bowel disease. Nature 491, 119-124 (2012).

96. McGovern, D.P. et al. Genome-wide association identifies multiple ulcerative colitis susceptibility loci. Nat. Genet. 42, 332-337 (2010).

97. Barrett, J.C. et al. Genome-wide association defines more than 30 distinct susceptibility loci for Crohn's disease. Nat. Genet. 40, 955-962 (2008).

98. Rioux, J.D. et al. Genome-wide association study identifies new susceptibility loci for Crohn disease and implicates autophagy in disease pathogenesis. Nat Genet 39, 596-604 (2007).

99. Ogura, Y. et al. A frameshift mutation in NOD2 associated with susceptibility to Crohn's disease. Nature 411, 603-606 (2001).

100. Hampe, J. et al. A genome-wide association scan of nonsynonymous SNPs identifies a susceptibility variant for Crohn disease in ATG16L1. Nat. Genet. 39, 207-211 (2007).

101. Gutierrez, M.G., Master, S.S., Singh, S.B., Taylor, G.A., Colombo, M.I. \& Deretic, $V$. Autophagy is a defense mechanism inhibiting BCG and Mycobacterium tuberculosis survival in infected macrophages. Cell 119, 753-766 (2004).

102. Cadwell, K. Crosstalk between autophagy and inflammatory signalling pathways: balancing defence and homeostasis. Nat. Rev. Immunol. 16, 661-675 (2016).

103. Plantinga, T.S. etal. Crohn's disease-associated ATG16L1 polymorphism modulates pro-inflammatory cytokine responses selectively upon activation of NOD2. Gut 60, 1229-1235 (2011).

104. Cadwell, K. et al. A key role for autophagy and the autophagy gene Atg16l1 in mouse and human intestinal Paneth cells. Nature 456, 259-263 (2008).

105. Deuring, J.J. et al. Genomic ATG16L1 risk allele-restricted Paneth cell ER stress in quiescent Crohn's disease. Gut 63, 1081-1091 (2014).

106. Hosomi, S., Kaser, A. \& Blumberg, R.S. Role of endoplasmic reticulum stress and autophagy as interlinking pathways in the pathogenesis of inflammatory bowel disease. Curr. Opin. Gastroenterol. 31, 81-88 (2015).

107. Tschurtschenthaler, M. et al. Defective ATG16L1-mediated removal of IRE1alpha drives Crohn's disease-like ileitis. J. Exp. Med. 214, 401-422 (2017).

108. Chu, H. et al. Gene-microbiota interactions contribute to the pathogenesis of inflammatory bowel disease. Science 352, 1116-1120 (2016).

109. Wildenberg, M.E. et al. The ATG16L1 risk allele associated with Crohn's disease results in a Rac1-dependent defect in dendritic cell migration that is corrected by thiopurines. Mucosal Immunol. 10, 352-360 (2017).

110. Boada-Romero, E., Serramito-Gomez, I., Sacristan, M.P., Boone, D.L., Xavier, R.J. \& Pimentel-Muinos, F.X. The T300A Crohn's disease risk polymorphism impairs function of the WD40 domain of ATG16L1. Nat. Commun. 7, 11821 (2016).

111. Zhu, X. et al. Cytosolic HMGB1 controls the cellular autophagy/apoptosis checkpoint during inflammation. J. Clin. Invest. 125, 1098-1110 (2015).

112. Diamanti, M.A. et al. IKKalpha controls ATG16L1 degradation to prevent ER stress during inflammation. JExp. Med. 214, 423-437 (2017).

113. Lahiri, A., Hedl, M. \& Abraham, C. MTMR3 risk allele enhances innate receptor-induced signaling and cytokines by decreasing autophagy and increasing caspase-1 activation. Proc. Natl Acad. Sci. USA 112, 10461-10466 (2015).

114. Odendall, C. et al. Diverse intracellular pathogens activate type III interferon expression from peroxisomes. Nat. Immunol. 15, 717-726 (2014).

115. Terawaki, S. et al. RUN and FYVE domain-containing protein 4 enhances autophagy and lysosome tethering in response to interleukin-4. J. Cell Biol. 210, 1133-1152 (2015).

116. Huttlin, E.L. et al. The BioPlex network: a systematic exploration of the human interactome. Cell 162, 425-440 (2015).

117. Zurek, B. et al. TRIM27 negatively regulates NOD2 by ubiquitination and proteasomal degradation. PLOS ONE 7, e41255 (2012). 
118. Wang, C.Y. et al. ZIP8 is an iron and zinc transporter whose cell-surface expression is up-regulated by cellular iron loading. J. Biol. Chem. 287, 34032-34043 (2012).

119. Xie, X. et al. Molecular basis of ubiquitin recognition by the autophagy receptor CALCOCO2. Autophagy 11, 1775-1789 (2015).

120. Kim, B.W., Kwon do, H. \& Song, H.K. Structure biology of selective autophagy receptors. BMB Rep 49, 73-80 (2016).

121. Lahiri, A. \& Abraham, C. Activation of pattern recognition receptors upregulates metallothioneins, thereby increasing intracellular accumulation of zinc, autophagy, and bacterial clearance by macrophages. Gastroenterology 147, 835-846 (2014).

122. Li, D. et al. A pleiotropic missense variant in SLC39A8 is associated with Crohn's disease and human gut microbiome composition. Gastroenterology 151, 724-732 (2016).

123. Franco, L.H. et al. The ubiquitin ligase Smurf1 functions in selective autophagy of Mycobacterium tuberculosis and anti-tuberculous host defense. Cell Host Microbe 21, 59-72 (2017).

124. Coly, P.M. et al. Chemotactic G protein-coupled receptors control cell migration by repressing autophagosome biogenesis. Autophagy 12 , 2344-2362 (2016).

125. Ejlerskov, P. et al. Tubulin polymerization-promoting protein (TPPP/ p25alpha) promotes unconventional secretion of alpha-synuclein through exophagy by impairing autophagosome-lysosome fusion. J. Biol. Chem. 288, 17313-17335 (2013).

126. Xu, D. et al. Modification of BECN1 by ISG15 plays a crucial role in autophagy regulation by type I IFN/interferon. Autophagy 11, 617-628 (2015).

127. Bertolo, C. et al. LITAF, a BCL6 target gene, regulates autophagy in mature B-cell lymphomas. Br. J. Haematol. 162, 621-630 (2013).

128. Stappenbeck, T.S. et al. Crohn disease: a current perspective on genetics, autophagy and immunity. Autophagy 7, 355-374 (2011).
129. Singh, S.B., Davis, A.S., Taylor, G.A. \& Deretic, V. Human IRGM induces autophagy to eliminate intracellular mycobacteria. Science $\mathbf{3 1 3}$, 1438-1441 (2006).

130. Chauhan, S., Mandell, M.A. \& Deretic, V. IRGM governs the core autophagy machinery to conduct antimicrobial defense. Mol. Cell 58, 507-521 (2015).

131. Mogi, C. et al. Involvement of proton-sensing TDAG8 in extracellular acidification-induced inhibition of proinflammatory cytokine production in peritoneal macrophages. J. Immunol. 182, 3243-3251 (2009).

132. Hazzan, D. et al. Crohn's-like colitis, enterocolitis and perianal disease in Hermansky-Pudlak syndrome. Colorectal Dis. 8, 539-543 (2006).

133. Schwerd, T. et al. Impaired antibacterial autophagy links granulomatous intestinal inflammation in Niemann-Pick disease type C1 and XIAP deficiency with NOD2 variants in Crohn's disease. Gut; e-pub ahead of print (2016).

134. Akbar, M.A., Mandraju, R., Tracy, C., Hu, W., Pasare, C. \& Kramer, H. ARC Syndrome-linked $\mathrm{Vps} 33 \mathrm{~B}$ protein is required for inflammatory endosomal maturation and signal termination. Immunity 45, 267-279 (2016).

135. Jiang, P. \& Mizushima, N. Autophagy and human diseases. Cell Res 24, 69-79 (2014).

136. Hasegawa, J., Iwamoto, R., Otomo, T., Nezu, A., Hamasaki, M. \& Yoshimori, T. Autophagosome-lysosome fusion in neurons requires INPP5E, a protein associated with Joubert syndrome. EMBO J. 35, 1853-1867 (2016).

137. Lee, M.Y. et al. Peroxisomal protein PEX13 functions in selective autophagy. EMBO Rep. 18, 48-60 (2016).

138. Kaizuka, T. et al. An autophagic flux probe that releases an internal control. Mol. Cell 64, 835-849 (2016).

139. Yoshii, S.R. et al. Systemic analysis of Atg5-null mice rescued from neonatal lethality by transgenic ATG5 expression in neurons. Dev. Ce/l 39, 116-130 (2016) 